\title{
Magnetocaloric Cooling Device with Reciprocating Motion of the Magnetic Field Source
}

\author{
R. Kolano, A. Kolano-Burian, M. Hreczka, M. Polak, J. Szynowski and W. Tomaka \\ Instytut Metali Nieżelaznych w Gliwicach, Zakład Materiałów Funkcjonalnych, Gliwice, Poland
}

(Received March 30, 2016)

This paper describes the magnetic cooling device model designed and built at the Institute of Non-Ferrous Metals in Gliwice, which utilizes reciprocating motion of a magnetic field source and a cyclic flow of a coolant through regenerator. The regenerator made from gadolinum made it possible to obtain an adiabatic temperature change of $2.5 \mathrm{~K}$ at the magnetic field of $0.8 \mathrm{~T}$. The magnetic field source was built using neodymium magnets according to our own technology. For the heat transfer, a liquid having the specific heat of about $4000 \mathrm{~J} /(\mathrm{kg} \mathrm{K})$ has been applied. We have also developed and applied a special driving system enabling reciprocating motion of the magnetic field source and of the pistons in the hot and cold heat exchangers. The tests were made with this cooling model showing that it was possible to obtain, at the frequency of $0.5 \mathrm{~Hz}$, the liquid temperature gradient of $4 \mathrm{~K}$. The potential energy savings resulting from application of this device have been evaluated and compared with the literature data presenting a pre-industrial prototype of the magnetic refrigerator utilizing similar solutions to those applied in our cooling model.

DOI: 10.12693/APhysPolA.129.1205

PACS/topics: $75.30 . \mathrm{Sg}, 75.50 . \mathrm{Ww}$

\section{Introduction}

There is a great interest in the last two decades in the research on magnetic refrigeration, which is related with recent achievements in materials science and development of new technologies making it possible to obtain practically useful cooling parameters. Magnetic refrigeration is a cooling technology utilizing the magnetocaloric effect, which is a fundamental property of ferromagnetic materials and is accompanied by adiabatic changes of their temperature and isothermal changes of entropy under the influence of an external magnetic field. The research on magnetic cooling was recently undertaken worldwide under many national and international projects. One of the key European projects in this subject area entitled "Solid State Energy Efficient Cooling" (SSEEC) was performed in the years 2008-2011 by an international consortium composed of the leading European research centres and industrial organisations, with a goal to advance magnetic refrigeration to a stage where it can be considered for commercial application [1, 2]. Although the project brought many interesting results, including new magnetic refrigerants and three prototype cooling engines utilizing the optimal materials developed, the findings from this projects can hardly be referred to as anticipated breakthrough in the technology of magnetic cooling. Some recent analyses indicate that it may still take over twenty years of intensive research and development activity, leading to significant progress in magnetocaloric materials and design concepts, so as to enable commercialisation of the magnetic cooling and make it competitive with the conventional vapour compression based technology [3]. Up to the present, over 40 advanced prototypes of magnetic coolers have been built worldwide but it is difficult to find in the literature reliable experimental data needed to compare efficiency of magnetic refrigeration with conventional vapour compression based technology. It is also necessary to conduct intensive search for relatively inexpensive permanent magnets and superconductors for magnetic field sources.

In the work [4], Okamura et al. demonstrated a rotating-type magnetic refrigerator utilizing cylindrical magnetic field source, $27 \mathrm{~cm}$ in an outer diameter and $40 \mathrm{~cm}$ long, generating a magnetic field of $0.77 \mathrm{~T}$. The regenerator was made from the GdDy alloy, and a special pump was used for circulation of the heat transfer fluid (water). A maximum cooling power obtained was $60 \mathrm{~W}$. The authors improved later their refrigerator by increasing magnetic field intensity to $0.9 \mathrm{~T}$ and by application of a new refrigerator from gadolinium so that the cooling efficiency of the device increased to $100 \mathrm{~W}$ [5]. Mueller et al. developed and built a pre-industrial prototype of magnetocaloric system consisting of a field source with flat poles and two regenerators made from gadolinum inserts, $0.6 \mathrm{~mm}$ thick and $100 \mathrm{~mm}$ long [6]. The mass of the system is $34 \mathrm{~kg}$, temperature span between the cold sink and hot sink reaches $33^{\circ} \mathrm{C}$, and the cooling power is $110 \mathrm{~W}$. In the paper [7], a reciprocating magnetic refrigerator incorporating two Halbach-type magnetic field sources and a regenerator made of $1 \mathrm{~mm}$ thick gadolinum inserts separated by a distance of $30 \mathrm{~mm}$, has been described. Mass of the regenerator is $400 \mathrm{~g}$, and the operating frequency - $0.5 \mathrm{~Hz}$. After several cycles of refrigeration, the maximum temperature span achieved between cold and hot ends was about $12{ }^{\circ} \mathrm{C}$, and a cooling power ranged from 80 to $100 \mathrm{~W}$. In a work [8], complex studies on magnetocaloric effect combined with the construction of prototypes of the magnetic cooling devices have been presented. A small-scale magnetocaloric cooling device, designed as a push-pull system with two permanent magnets with a field of about $0.85 \mathrm{~T}$, has been presented in the paper [9]. The device was constructed so as to 
make it possible to change some parameters of the cooling process and to change type of a working material and the design of heat exchangers. The measurements were performed using $35 \mathrm{~g}$ of gadolinium as a working material and ethanol as a heat transfer liquid, showing that the maximum temperature span at a room temperature was $3.1 \mathrm{~K}$.

Experimental works aimed at building models of the magnetic cooling devices were also conducted in Poland, at the Wrocław University of Technology [10].

In this paper, a prototype model of a magnetic cooling device designed and built at Institute of Non-Ferrous Metals (IMN), utilizing reciprocating motion of the magnetic field source and cyclic flow of a liquid ensuring suitable heat transfer, has been presented. The results of the performance tests carried out for this model have been discussed.

\section{Design of a model of magnetic cooling device}

The cooling effect takes place in magnetocaloric refrigerators as a result of cyclic magnetization and demagnetization of a ferromagnetic material, i.e. regenerator (Fig. 1a). In the magnetization phase $(H>0)$, spin orientation becomes ordered so that regenerator's entropy decreases by $\Delta S_{m}$ (Fig. 1b) in reference to that in demagnetization stage $(H=0)$. Under constant pressure, the value of entropy $S_{m}$ of magnetocaloric material depends both on magnetic field $H$ and on temperature $T$. The cooling effect is accompanied by two processes: in the first of them isothermal magnetization takes place, and in the second - adiabatic demagnetization (point $D$ in Fig. 1b), during which the temperature decreases from $T_{1}$ to $T_{0}$.

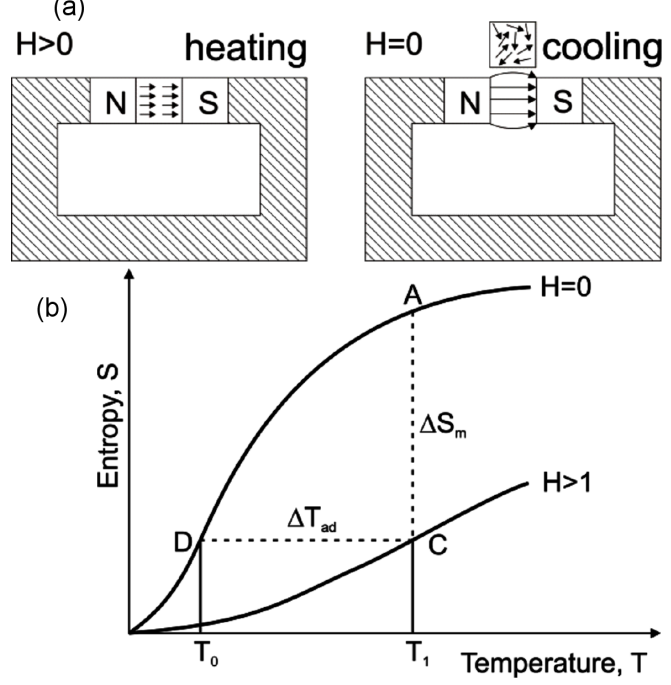

Fig. 1. Principle of magnetocaloric effect: (a) magnetisation of a material resulting in its heating and demagnetisation causing its cooling, (b) dependence of the magnetic entropy $S_{m}$ on temperature for two different values of the magnetic field $H$.

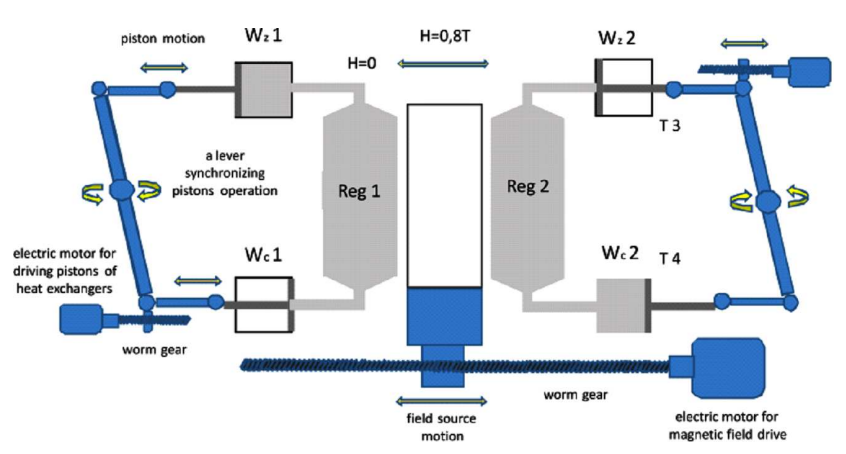

Fig. 2. Design and principle of operation of a cooling device with reciprocating motion of a magnetic field source and cyclic flow of a liquid through regenerators Reg1 and Reg2.

In order to practically utilize the magnetocaloric effect in the cooling devices it is necessary to develop a technical method for separation and then storing of the thermal effects taking place in a regenerator during its cyclic magnetization and demagnetization. During designing and building of such devices it is necessary to solve many problems requiring interdisciplinary expertise in the field of electrical drives, electronic control, materials engineering, thermodynamics, hydrodynamics, and others. A diagram of the cooling model with reciprocating motion of magnetic field source and cyclic flow of a liquid through regenerators is shown in Fig. 2.

The model consists of the following components:

- two regenerators Reg1 and Reg2 from gadolinum,

- magnetic field source together with its drive,

- two cold sinks (Wz1 and Wz2) and two hot sinks (Wc1 and Wc2),

- synchronous drives of the pistons T1, T2, T3, and T4 of heat exchangers, and

- control system of the cooling device.

\section{Description of main subassemblies 3.1. Regenerator}

Two almost similar regenerators have been applied as the model cooling device. A photograph of the regenerator is shown in Fig. 3 (two Gd segments with con-

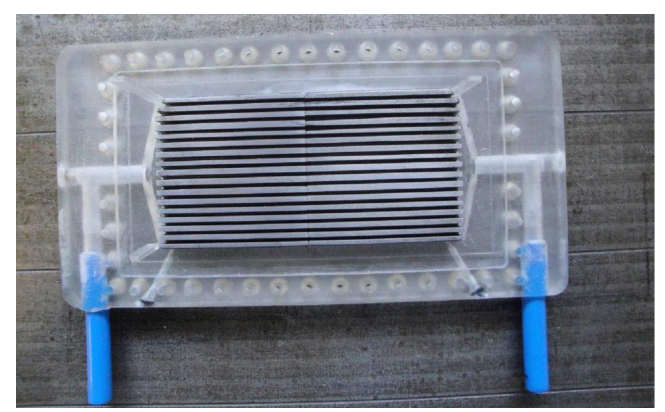

Fig. 3. Photograph of the gadolinum regenerator placed in a tightly closed container with visible inlet and outlet of a liquid. 
nected surfaces, placed in a tightly closed container), whereas Fig. 4 shows the characteristics MCE $=f(T)$ for gadolinum, from which the regenerators were made.

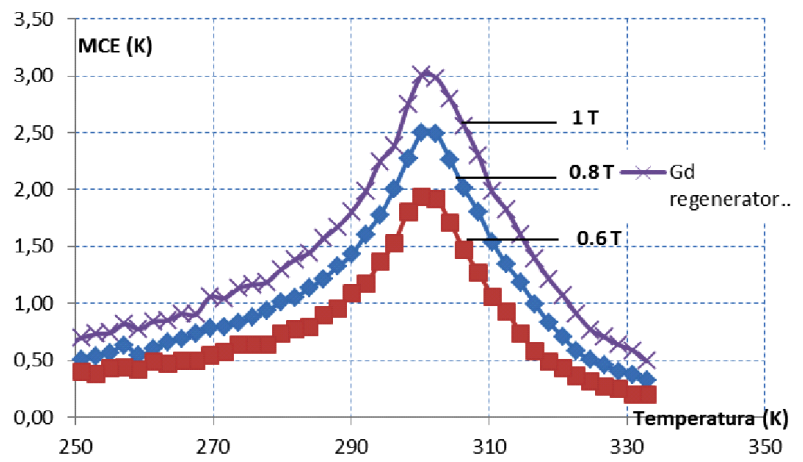

Fig. 4. Dependence of magnetocaloric effect (MCE) on temperature for gadolinum regenerator.

The gadolinum properties are the following: mass density - $7.9 \mathrm{~g} / \mathrm{cm}^{3}$, specific heat $c_{p}=230 \mathrm{~J} /(\mathrm{kg} \mathrm{K})$, thermal conductivity $\lambda=10.5 \mathrm{~W} /(\mathrm{m} \mathrm{K})$, mass of a regenerator - $369 \mathrm{~g}$, dimensions: $10 \times 50 \times 12 \mathrm{~mm}^{3}$, thickness of an insert $-2.2 \mathrm{~mm}$, distance between the inserts $-1.2 \mathrm{~mm}$

\subsection{Magnetic field source}

The magnetic field source (Fig. 5) consists of four NdFeB magnets (numbered 2, 4, 6, and 8) with the dimensions of $25 \times 65 \times 50 \mathrm{~mm}^{3}$ and of two magnets (3 and 7), $100 \times 40 \times 50 \mathrm{~mm}^{3}$ in dimensions, and also of five cuboids from armco iron, which are the constructional and screening elements. The role of magnetic poles is played by two cuboids from armco iron, $100 \times 25 \times 50 \mathrm{~mm}^{3}$ in dimensions.

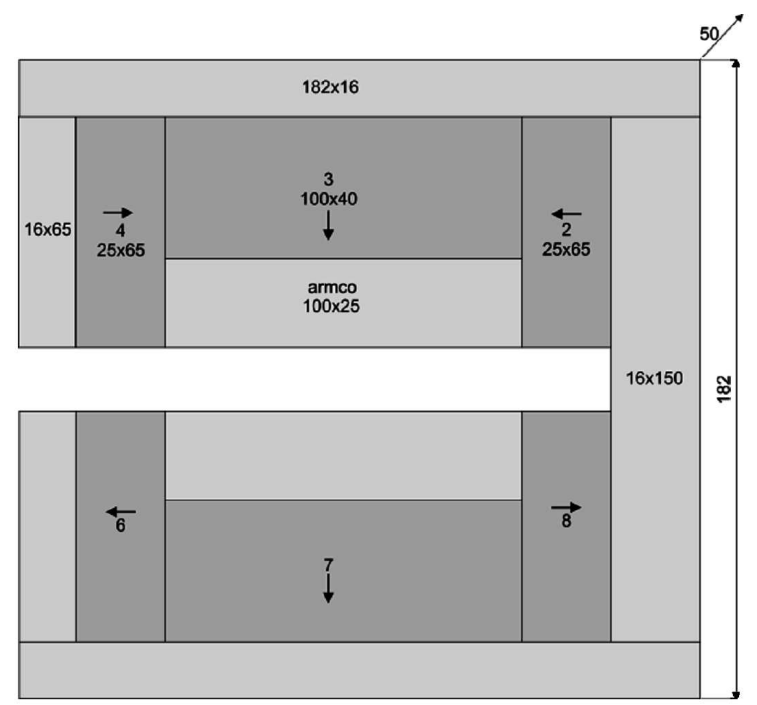

Fig. 5. Schematic diagram of a magnetic field source.

The parameters of the magnetic field source built are the following:

- gap dimensions $($ length $\times$ height $\times$ width): $100 \times$ $20 \times 50 \mathrm{~mm}^{3}$,

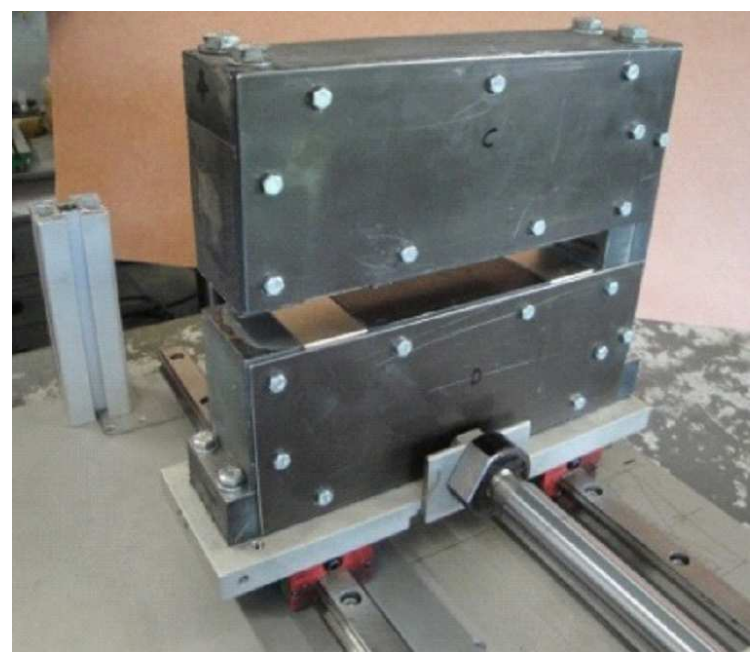

Fig. 6. Photograph of a magnetic field source.

- magnetic field in a gap: $0.8 \mathrm{~T}$,

- outer dimensions: $182 \times 182 \times 50 \mathrm{~mm}^{3}$,

- mass: $8.5 \mathrm{~kg}$.

Properties of a liquid used for the transfer of heat related with the changes of regenerator's temperature were the following:

- specific density: $1.07 \mathrm{~g} / \mathrm{cm}^{3}$,

- working temperature: from $40^{\circ} \mathrm{C}$ to $-20^{\circ} \mathrm{C}$,

- specific heat: about $4000 \mathrm{~J} /(\mathrm{kg} \mathrm{K})$,

- thermal conductivity $\lambda$ : about $0.6 \mathrm{~W} /(\mathrm{m} \mathrm{K})$.

\subsection{Heat exchangers}

Figure 7 shows schematic diagram of a heat exchanger; its parameters being:

- active mass: $66 \mathrm{~g}$,

- density of $\mathrm{Cu}: 8.9 \mathrm{~g} / \mathrm{cm}^{3}$,

- thermal conductivity of $\mathrm{Cu}: 400 \mathrm{~W} /(\mathrm{m} \mathrm{K})$,

- specific heat of $\mathrm{Cu}: 385 \mathrm{~J} /(\mathrm{kg} \mathrm{K})$.

The design shown in Fig. 7 had both the hot heat exchangers and cold heat exchangers.

\section{Description of operation}

The applied driving systems of the magnetic field source, pistons of heat exchangers and the control system of the cooling device model made it possible to conduct the cooling process in accordance with the schematic diagram shown in Fig. 8. In order to simplify the description of model operation, only regenerator Reg1 will be taken 


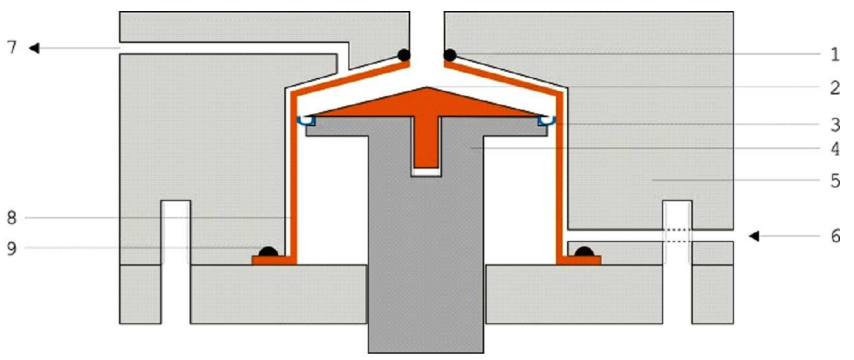

Fig. 7. Design of heat exchanger $(1-$ seal of a copper cylinder, 2 - copper cone, 3 - piston seal, 4 - piston, 5 - plastic casing, 6 - cooling liquid inlet, 7 - cooling liquid outlet, 8 - copper cylinder, 9 - cylinder seal).

into account in this description. It should be noted that the same cooling stages take place in both regenerators, i.e. Reg1 and Reg2, but with a time delay of about $1.5 \mathrm{~s}$.

In a starting position, the magnetic field source is situated between Reg1 and Reg2, and the cold heat sink Wz1 is filled with a liquid, whereas the hot heat sink is empty. In the first stage of operation, the field source moves towards the regenerator Reg1 causing its magnetisation and heating. In the second stage, a liquid is transferred from Wz1 to Reg1 so that it replaces a liquid heated earlier, which in turn is transported to hot heat sink Wc1 where the heat is returned to the environment. Under the stage 3, the heat is still transferred from $\mathrm{Wc1}$ to the environment, whereas magnetic field is being removed from Reg1, which results in cooling down of the latter. In the stage 4 , the liquid is transported from Wc1 to Reg1 so that the cooled down liquid from Reg1 gets to Wz1, where it takes heat from the cold sink (the latter then cools down). At this stage, a temperature gradient between the beginning and end of the regenerator is already observed, and this phenomenon will deepen in the further stages.

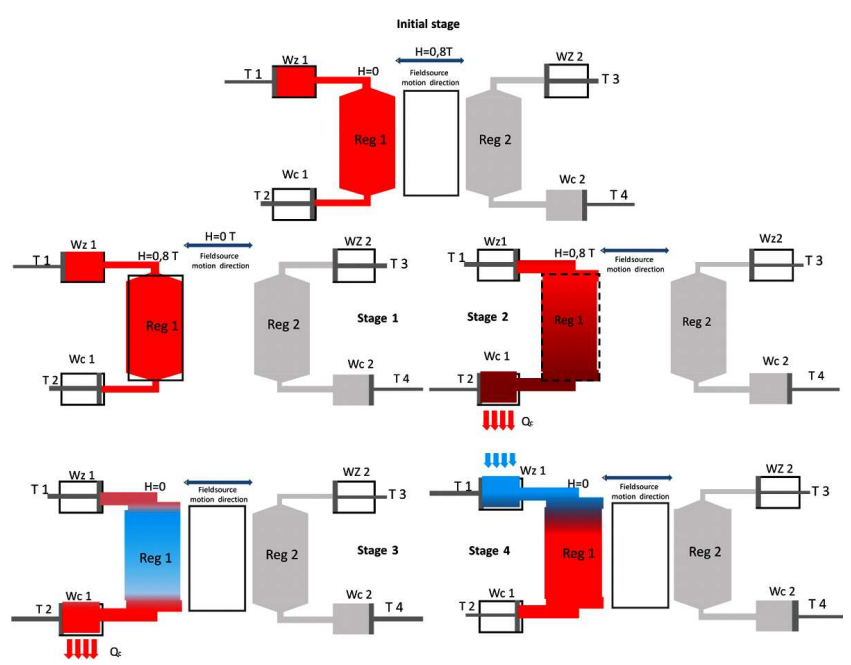

Fig. 8. The stages of cooling with the use of magnetocaloric effect of gadolinum.

\section{Cooling performance evaluation}

Figure 9 shows a photo of the developed cooling system model with reciprocating motion of a magnetic field source.

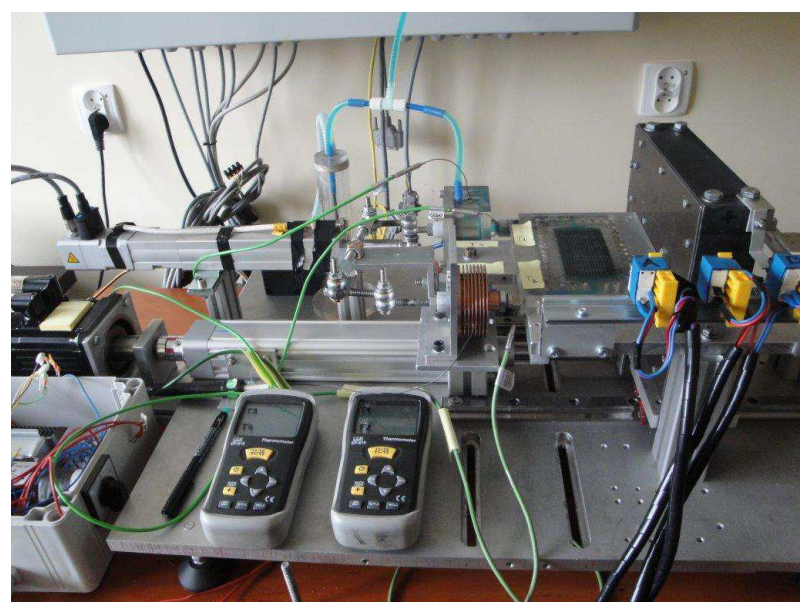

Fig. 9. Photograph of a cooling system developed.

The model was subjected to performance tests, which included the check for operation of the magnetic field source drive, and the drives of pistons of heat exchangers. It was found that the maximum speed of a field source is $5 \mathrm{~m} / \mathrm{s}$, which means that the shortest time necessary for that source to approach the regenerator is $0.01 \mathrm{~s}$ (the width of a regenerator and width of a field source are $50 \mathrm{~mm}$ ). The applied drive of the pistons of heat exchangers makes it possible to obtain the frequency up to $1 \mathrm{~Hz}$. The tests were carried out at the frequency of $0.5 \mathrm{~Hz}$. Temperature of a liquid was measured using thermocouples in the regenerator's hot and cold ends. It was found that in case of the initial temperature of the cooling model equal to $22^{\circ} \mathrm{C}$, the temperature of a liquid after 10 cycles of magnetization and demagnetization increased to $24^{\circ} \mathrm{C}$ near the hot end of regenerator and decreased to $20^{\circ} \mathrm{C}$ near the cold end. That means that temperature gradient of the liquid between hot end and cold end of the regenerator reaches about $4{ }^{\circ} \mathrm{C}$.

TABLE I

Comparison of the parameters of our model with those of the prototype from the reference [6].

\begin{tabular}{c|c|c}
\hline \hline Parameter & $\begin{array}{c}\text { IMN } \\
\text { model }\end{array}$ & $\begin{array}{c}\text { Prototype } \\
{[6]}\end{array}$ \\
\hline $\begin{array}{c}\text { magnetic field in a gap } \\
\text { of the field source }\end{array}$ & $0.8 \mathrm{~T}$ & $1.6 \mathrm{~T}$ \\
adiabatic temperature change $\Delta T_{\mathrm{ad}}$ & $2.5 \mathrm{~K}$ & $4.2 \mathrm{~K}$ \\
regenerator insert thickness & $2.2 \mathrm{~mm}$ & $0.6 \mathrm{~mm}$ \\
distance between inserts & $1.2 \mathrm{~mm}$ & $0.4 \mathrm{~mm}$ \\
active surface area of regenerator & $374 \mathrm{~cm}^{2}$ & $\mathrm{~N} / \mathrm{A}$ \\
frequency & $0.5-1 \mathrm{~Hz}$ & $0.5-2 \mathrm{~Hz}$ \\
amount of liquid in regenerator & $26 \mathrm{~cm}^{3}$ & $\mathrm{~N} / \mathrm{A}$
\end{tabular}


Although the tests confirmed the existence of magnetocaloric effect in our model of a cooling device, its cooling efficiency was clearly lower than that of the prototype described in the work [6]. The parameters having the greatest impact on this efficiency are listed in Table I. It is seen that our model works at twice lower magnetic field than the referenced prototype and, consequently, the adiabatic temperature change $\Delta T_{\mathrm{ad}}$ is also almost twice smaller.

\section{Summary and conclusions}

The magnetic cooling device model utilizing reciprocating motion of a magnetic field source and a cyclic flow of a liquid through regenerator has been designed and built at the Institute of Non-Ferrous Metals in Gliwice. The regenerator, $100 \mathrm{~mm}$ in length, was made from $2.2 \mathrm{~mm}$ thick gadolinum plates. The adiabatic temperature change $\Delta T_{\mathrm{ad}}$ was $2.5 \mathrm{~K}$ for the magnetic field $H=0.8 \mathrm{~T}$.

The magnetic field source was built using neodymium magnets and IMN's own technology; dimensions of the gap being: length $L=100 \mathrm{~mm}$, width $w=50 \mathrm{~mm}$, height $h=20 \mathrm{~mm}$. Magnetic field in the gap is $0.8 \mathrm{~T}$. For the heat transfer, a liquid having the specific heat of about $4000 \mathrm{~J} /(\mathrm{kg} \mathrm{K})$ has been applied. A special driving system enabling reciprocating motion of the magnetic field source and of the pistons in the hot and cold heat exchangers, moving with the speed up to $5 \mathrm{~m} / \mathrm{s}$, has been developed and applied. Operation of the model cooling device is controlled by the dedicated microprocessor system.

The cooling device developed at IMN makes it possible to obtain, at the frequency $f=0.5 \mathrm{~Hz}$, the liquid temperature gradient reaching $4 \mathrm{~K}$. The potential energy savings resulting from application of this device have been evaluated based on the data given in a work [6], presenting a pre-industrial prototype of the magnetic refrigerator utilizing similar solutions to those applied in our cooling model. The energy efficiency analysis was made for the reference prototype and its coefficient of performance (COP) was determined, showing that it would be possible to achieve the value of COP of 5 or even greater provided that better magnetocaloric materials and stronger magnetic field source are applied, and further constructional improvements are introduced so as to increase operating frequency of the device. It was found that the potential electric energy savings might reach $50 \%$.
Despite the fact that the studies on magnetic refrigeration have already been conducted worldwide for over 20 years, the research on this topic is still in its initial stage so that comparing energy efficiency of the magnetic cooling and conventional vapour compression based cooling is still extremely difficult. Over 40 advanced working prototypes have been built worldwide but no reliable data on their energy efficiency are available. An important indication on potentially high energy savings offered by the novel technology is the fact that the efficiency of magnetic refrigeration may theoretically reach $30-60 \%$ of the Carnot cycle, whereas this efficiency is equal to only $5-10 \%$ in case of the conventional cooling.

\section{Acknowledgments}

Authors are thankful for the financial support from the National Centre for Research and Development in Poland within the framework of PBS grant No. PBS2/A5/36/2013.

\section{References}

[1] Solid State Energy Efficient Cooling SSEEC.

[2] SSEEC FP\%-NMP programme, project reference No. 214864, Final Report.

[3] J.S. Brown, P.A. Domanski, Appl. Therm. Eng. 64, 252 (2014).

[4] T. Okamura, K. Yamada, N. Hirano, S. Nagaya, Int. J. Refrigerat. 29, 1327 (2006).

[5] T. Okamura, K. Yamada, N. Hirano, S. Nagaya, in: Proc. Second Int. Conf. on Magnetic Refrigeration at Room Temperature, Portoz (Slovenia), International Institute of Refrigeration, Paris 2007, p. 377.

[6] Ch. Mueller, C. Vasile, M. Risser, J.C. Heitzler, B. Keith, in: Int. Symp. on Next-Generation Air Conditioning and Refrigeration Technology, Tokyo (Japan), 2010.

[7] M. Balli, O. Sari, O. Mahmed, Ch. Besson, Ph. Bonhote, D. Duc, J. Forchelet, in: Appl. En. 98, 556 (2012).

[8] J. Barbose, J. Lozano, P. Trevizoli, in: 15th Brazilian Congress of Thermal Sciences and Engineering, Belen PA (Brazil), 2014.

[9] J. Kastil, J. Tetek, A. Tucek, Acta Phys. Pol. A 124, 740 (2013).

[10] A. Czemuszewicz, J. Kaleta, M. Królewicz, D. Lewandowski, R. Mech, P. Wiewiórski, Int. J. Refrigerat. 37, 72 (2014). 